\title{
Occupational HIV infection: Can we talk?
}

\author{
LE NICOLLE, MD
}

$\mathrm{H}$

EALTH CARE WORKERS ARE AT RISK OF ACQUIRING human immunodeficiency virus (HIV) infection from their patients. This risk is exceedingly small, having been repeatedly measured at approximately 0.3 to $0.5 \%$ following significant needlestick/sharps exposure to HIV patients (1). Measurable risk for acquisition of HIV infection is associated only with needlestick and sharps exposures and, for needlestick, possibly only with hollow bore needles. In several prospective studies no quantifiable risk has been identified for mucous membrane or intact skin-blood contact (1). Occupational acquisition of HIV infection has not yet been reported in Canada (2), but the consistency of risk documented in other studies suggests it will occur.

There are, on the other hand, no reports of acquisition of HIV infection by patients from staff of health care institutions. About 5.4\% of cases of acquired immune deficiency syndrome (AIDS) in the United States have occurred in health care workers, including surgeons, anesthetists and other physicians engaged in invasive procedures (3). Thus, considerable patient exposure and potential for transmission should have occurred. Where patients of surgeons with HIV infection have been screened with HIV serology, no evidence for transmission to patients has been reported. Recently, however, a highly publicized case of possible dentist-to-patient transmission, despite the appropriate use of recommended precautions, has raised public concerns about the potential for acquisition of HIV from health care workers (4). The case reported is a controversial one and, despite thorough study, does not unequivocally document the suggested mode of transmission.

Current recommendations for the prevention of transmission of HIV in the workplace suggest that health care workers should follow universal precautions to avoid contact with potentially infectious blood $(5,6)$. These recommendations include care to avoid needlestick and other sharps injuries. It is recommended that health care workers who experience significant percutaneous or mucous membrane exposure to blood from any patient should undergo serological screening for potential bloodborne agents, including HIV, and be monitored with standardized follow-up. As part of this follow-up, source patients, when known, should be tested for HIV if informed consent is given. Current guidelines do not recommend that health care workers known to be HIV-infected have restricted patient contact, although it is suggested that, on arr individual basis, some limitations in the performance of invasive procedures may be appropriate.

Universal precautions are rational and have been helpful to institutions in developing occupational health programs to minimize transmission of bloodborne agents. They are, however, general guidelines rather than specific and, as more information becomes available, appear inconsistent in some cases. Much of the emphasis on and cost of universal precautions focuses on prevention of blood contact through the use of barrier precautions, including gloves, gowns and masks. The risk of infection acquisition is, however, associated with sharps injuries, and it is not clear how effective barrier precautions are in preventing sharps injuries. The most efficient use of resources to prevent occupational transmission may be to focus on sharps injury elimination programs rather than gloving for all blood contact. A second concern is the requirement for informed consent from the source patient before HIV testing. Some health care workers perceive this as an inap-

Correspondence and reprints: Dr LE Nicolle, MS-675-D, Health Science Centre, 820 Sherbrook Street, Winnipeg, Manitoba R3A 1R9. Telephone (204) 787-4655 
propriate bias in favour of the patient in a straightforward situation of occupational risk. Significant exposure to HIV may have substantial impact for the health care worker, including modification of sexual practices and decisions with respect to pregnancy or nursing. The potential administration of post exposure zidovudine (AZT) prophylaxis following significant occupational exposure is a further complicating issue (7). There is no documented evidence that AZT is effective post exposure prophylaxis and, in fact, increasing evidence through case reports $(8,9)$ and animal studies (10) suggests a lack of efficacy. AZT is a toxic and expensive agent, and should be used only if the source patient is documented to be HIV positive. Thus, there are compelling occupational health arguments for the routine screening of source patients following significant exposure. The argument against such screening is primarily one of patient individual rights, particularly with respect to confidentiality (11). While the argument that a negative test may be a false negative (and thus testing is not helpful) is sometimes raised, this situation is likely infrequent, and improving diagnostic technologies including antigen detection and polymerase chain reaction techniques obviate this concern. What is lacking in these discussions is information, particularly Canadian information, documenting how informed consent is obtained, how frequently informed consent is obtained prior to testing of source patients, and how frequently source patients refuse such testing.

The 'dental case' has further contributed to the complexity of this issue. There is currently no consensus regarding the appropriate response. Despite the absence of evidence for transmission of HIV infection from an infected health care worker to a patient in the hospital setting, the reported case has led to suggestions that HIV positive health care workers should have restricted patient contact. The American Academy of Orthopedic Surgeons, the Surgical Infection Society, the Society of Thoracic Surgeons, and the American College of Surgeons in the United States have all suggested that HIV-infected surgeons should not operate, despite the lack of evidence for operative transmission from surgeon to patient. If HIV-infected health care workers should be restricted from performing certain patient care activities, then it follows that health care workers performing such procedures should be screened for HIV infection. This reasoning starts us down a slippery slope of HIV screening for questionable indications, the bottom of which cannot be discerned. The emerging scenario has the rights of health care workers restricted despite the absence of epidemiological evidence of risk, while the rights of patients are affirmed despite an undisputed measurable risk of transmission.

Where do we go with this complex, evolving, and emotionally charged issue? First, we need to acknowledge that our approaches to HIV infection and transmission are still often determined by social and political expedience rather than medical and scientific data. Second, more information is needed. Further studies documenting the risks of occupational infection may be helpful, but studies of attitudes and behaviour, and of the most efficient use of resources for hospital programs to prevent institutional transmission of infection, are important priorities. Several studies are now ongoing in the United States and are beginning to be reported (12-15). These are defining the risks of HIV acquisition in specific situations and the types of occupational health programs that are most efficient, as well as answering specific questions such as how often source patients refuse to be tested following informed consent. The important information generated from these studies should facilitate reasoned and knowledgeable recommendations of appropriate practices and, perhaps, generate information that the public will accept. Canadianbased studies are still required. Finally, a dialogue which includes HIV patients, health care workers and the public seems appropriate to move towards some consensus of where the balance should lie between patient and health care worker rights in matters relating to occupational transmission of HIV.

\section{REFERENCES}

1. Gerberging JL. Current epidemiologic evidence and case reports of occupationally acquired HIV and other bloodborne diseases. Infect Control Hosp Epidemiol 1990;11:S558-62.

2. Health and Welfare Canada. Occupational exposure to the human immunodeficiency virus among health care workers in Canada. CDWR 1988; 14:197-200.

3. Centres for Disease Control. Update: Acquired immunodeficiency virus infection among health-care workers. MMWR 1988;37:229-34.

4. Centers for Disease Control. Possible transmission of human immunodeficiency virus to a patient during an invasive dental procedure. MMWR 1990;39:492.

5. Centers for Disease Control. Recommendations for prevention of HIV transmission in health care settings. MMWR 1987;36(Suppl 2):6-7.

6. Health and Welfare Canada. Recommendations for prevention of HIV transmission in health care settings. CDWR 1987;13(Suppl 3):1-10.

7. Centers for Disease Control. Public Health Service statement on management of occupational exposure to human immodeficiency virus, including consideration regarding zidovudine 
post-exposure use. MMWR 1990;39(No. RR-1):1-14

8. Lange JMA, Boucher CAB, Hollak CEM, et al. Failure of zidovudine prophylaxis after accidental exposure to HIV-1. N Engl J Med 1990;322:1375-7.

9. Looke DFM, Grove DI. Failed prophylactic zidovudine after needlestick injury. Lancet 1990;i: 1280 .

10. Schinaze RF, Anderson DC, Fultz P, McClure HM. Prophylaxis with antiretroviral agents in Rhesus macaques inoculated with simian

immunodeficiency virus. Atlanta: 30th Interscience Conference on Antimicrobial Agents and Chemotherapy 1990:962. (Abst)

11. Chateauvert M, Duffre A, Gilmore N. HIV antibody testing: CMA counselling guidelines. Can Med Assoc J 1990;143:827-8. (Lett)
12. Lowenfels AB, Wormser GP, Jain R. Frequency of puncture injuries in surgeons and estimated risk of HIV infection. Arch Surg 1989;124:1284-6.

13. Doebbeling BN, Wenzel RP. The direct costs of universal precautions in a teaching hospital. JAMA 1990;264:2083-7.

14. Tokars I, Marcus R, Culver DH, Bell DM. Blood contacts during surgical procedures. Atlanta: 30th Interscience Conference on Antimicrobial Agents and Chemotherapy 1990:958. (Abst)

15. Manzella J, Falk S, McConville J, Kellogg J. Impact of a human immunodeficiency virus counselor on rates of informed consent and counseling prior to HIV testing. Atlanta: 30th Interscience Conference on Antimicrobial Agents and Chemotherapy 1990:964. (Abst) 


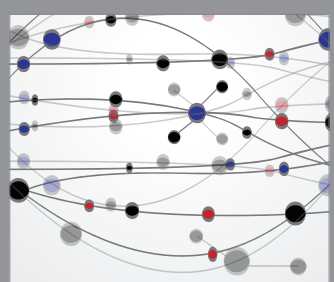

The Scientific World Journal
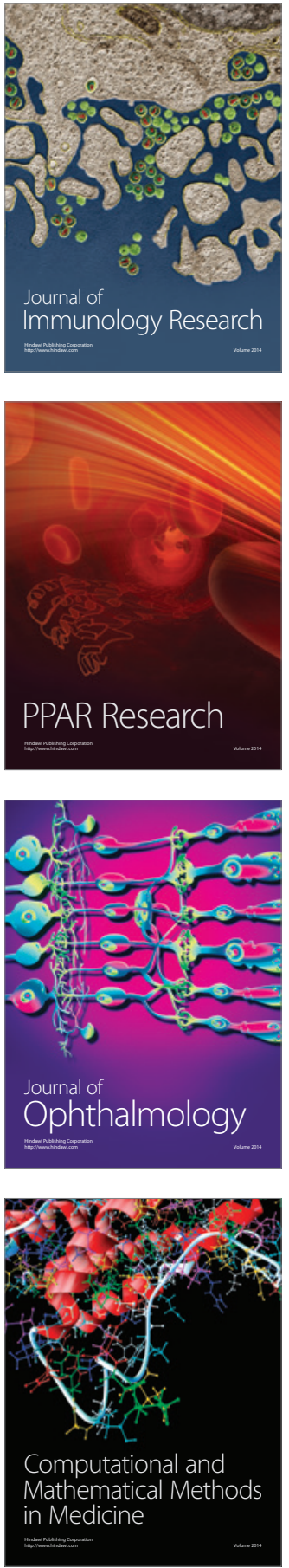

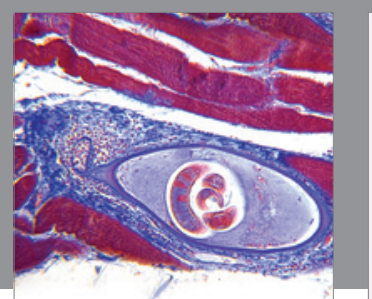

Gastroenterology Research and Practice

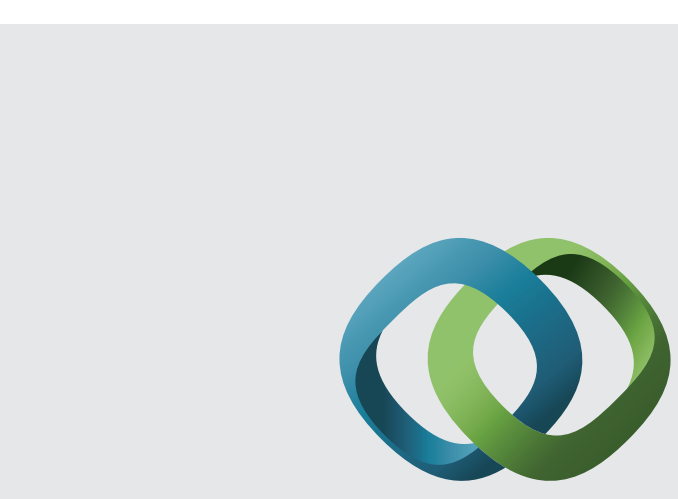

\section{Hindawi}

Submit your manuscripts at

http://www.hindawi.com
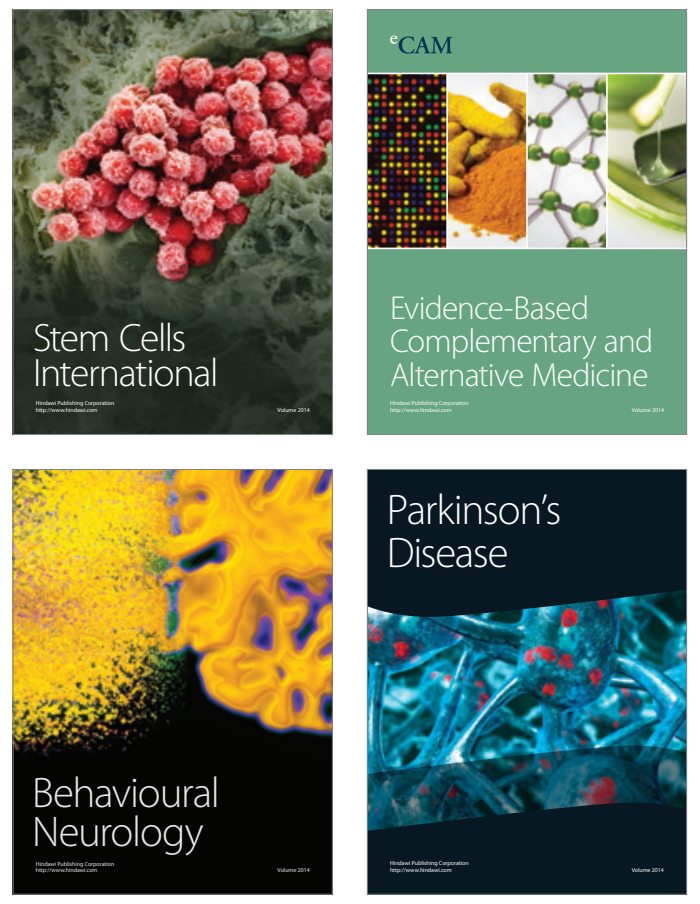
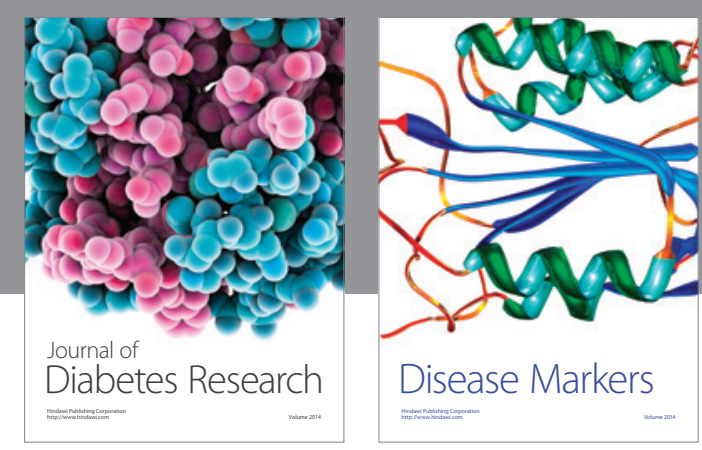

Disease Markers
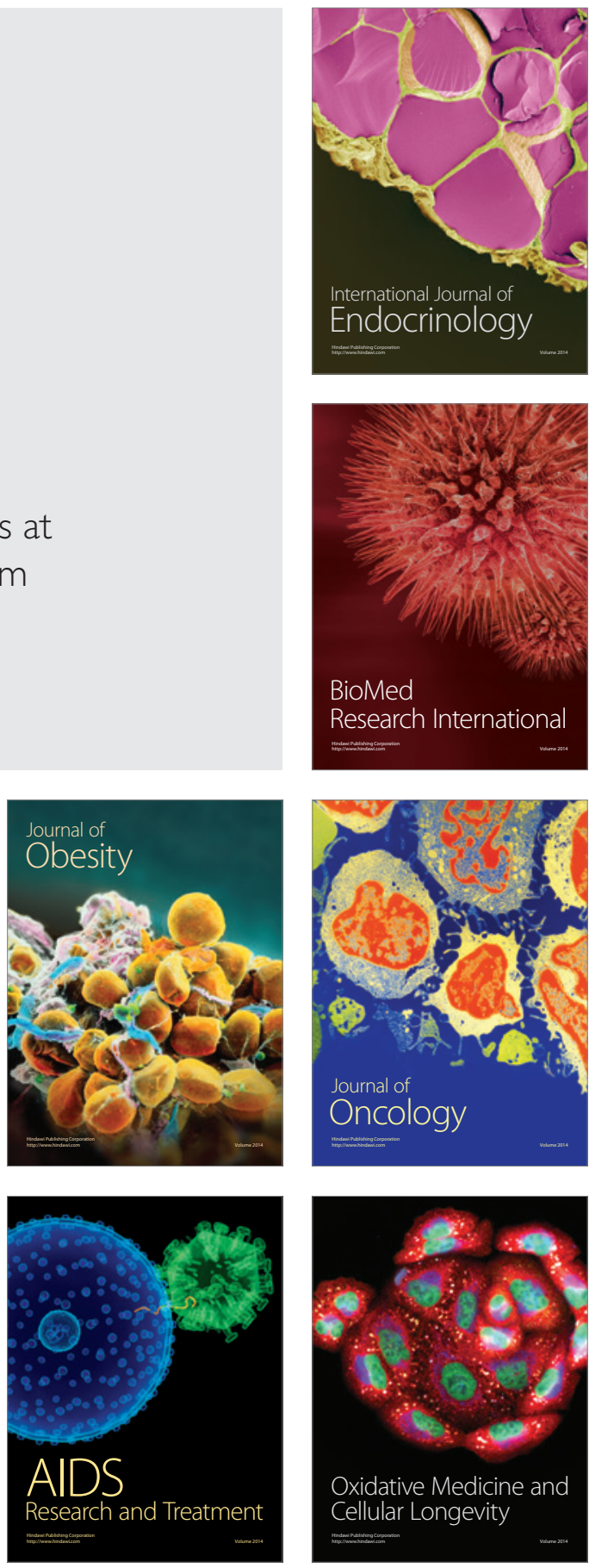